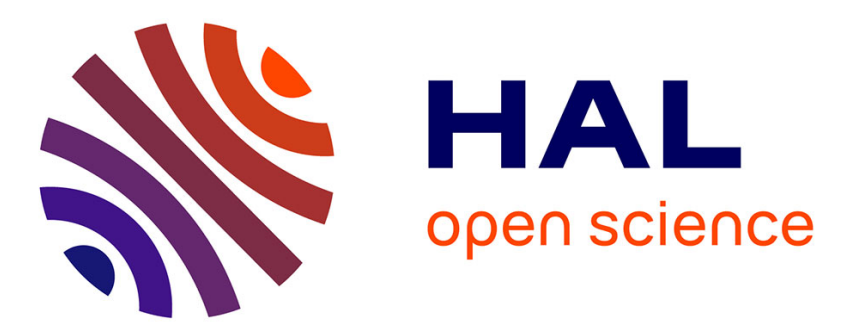

\title{
Magnétisme de films ultra-minces de Mn épitaxiés sur Fe(001) étudié en dichroïsme magnétique circulaire
}

\author{
S. Andrieu, M. Finazzi, F. Yubero, P. Arcade, F. Chevrier, H. Fischer, K.
} Hricovini, G. Krill, M. Piecuch

\section{- To cite this version:}

S. Andrieu, M. Finazzi, F. Yubero, P. Arcade, F. Chevrier, et al.. Magnétisme de films ultra-minces de Mn épitaxiés sur $\mathrm{Fe}(001)$ étudié en dichroïsme magnétique circulaire. Journal de Physique IV Proceedings, 1996, 06 (C7), pp.C7-117-C7-121. 10.1051/jp4:1996713 . jpa-00254501

\section{HAL Id: jpa-00254501 https://hal.science/jpa-00254501}

Submitted on 1 Jan 1996

HAL is a multi-disciplinary open access archive for the deposit and dissemination of scientific research documents, whether they are published or not. The documents may come from teaching and research institutions in France or abroad, or from public or private research centers.
L'archive ouverte pluridisciplinaire HAL, est destinée au dépôt et à la diffusion de documents scientifiques de niveau recherche, publiés ou non, émanant des établissements d'enseignement et de recherche français ou étrangers, des laboratoires publics ou privés. 


\title{
Magnétisme de films ultra-minces de Mn épitaxiés sur $\mathrm{Fe}(001)$ étudié en dichroïsme magnétique circulaire
}

\author{
S. Andrieu, M. Finazzi*, F. Yubero*, P. Arcade, F. Chevrier*, H. Fischer, K. Hricovini*, G. Krill* et \\ M. Piecuch \\ Laboratoire de Physique des Matériaux, URA du CNRS, Université Henri Poincaré Nancy 1 , \\ 54506 Vandouvre cedex, France \\ * LURE, CNRS/CEA, Université Paris-Sud, 91405 Orsay, France
}

\begin{abstract}
Résumé : Les propriétés magnétiques de films épitaxiés de Mn de 1 à 10 monocouches sur des couches tampons de $\mathrm{Fe} \mathrm{bcc} \mathrm{(001)} \mathrm{sont} \mathrm{étudiées} \mathrm{par} \mathrm{dichroïsme} \mathrm{magnétique} \mathrm{circulaire} \mathrm{en} \mathrm{X} \mathrm{mou.}$ Nous montrons tout d'abord que la croissance de $\mathrm{Mn}$ sur Fe(001) est bi-dimensionnelle et pseudomorphe au plan (001) du fer. Un ordre ferromagnétique est observé jusqu'à deux plans atomiques de $\mathrm{Mn}$ et le couplage interfacial entre les moments de $\mathrm{Mn}$ et Fe est ferromagnétique. Ce résultat est en désaccord avec les conclusions d'une expérience similaire récente. Nous montrons que cette différence provient de la préparation des échantillons.
\end{abstract}

\section{INTRODUCTION}

Grâce à l'Epitaxie par Jets Moléculaires (EJM), il est devenu possible de réaliser des hétéro-épitaxies de métaux, permettant ainsi d'explorer expérimentalement le domaine concernant les relations entre structure et magnétisme. En effet, si beaucoup de prévisions théoriques basées sur des calculs de bandes électroniques sont disponibles, les résultats d'un modèle à l'autre sont parfois contradictoires, et leur vérification expérimentale n'en est qu'à ces débuts. En outre, si on arrive à moduler les structures cristallographiques des métaux en EJM, les épaisseurs critiques de relaxation de ces structures sont souvent très faibles. Les quantités de matériaux disponibles (quelques plans atomiques) sont donc souvent trop petites pour les caractérisations magnétiques classiques. Cependant, une technique révolutionnaire de mesure magnétique est apparue récemment, appelé le dichroïsme magnétique circulaire de rayons X. Ainsi, les propriétés magnétiques de dépôts jusqu'à quelques fractions de monocouche sont accessibles par cette technique.

Expliquons en quelques mots le principe de cette technique. Lorsqu'un rayonnement de longueur d'onde appropriée interagit avec un atome, des transitions électroniques entre niveaux d'énergie s'effectuent. Si le rayonnement incident est polarisé circulairement, toutes les transitions entre niveaux de l'atome ne sont pas permises, à cause des règles de sélection dipolaire [1]. Ainsi, les probabilités de transition des spins up et down ne sont pas identiques en polarisation circulaire droite et gauche. Moyennant un traitement théorique qui conduit aux règles dites de somme [2], il est possible de calculer les moments orbital et de spin de l'atome sondé. Les avantages de cette technique sont donc multiples, à savoir, la grande sensibilité $(\approx 0,1$ monocouche), la sélectivité atomique, la détermination de l'orientation du moment, et la séparation des moments $\left\langle\mathrm{S}_{\mathrm{z}}\right\rangle$ et $\left\langle\mathrm{L}_{\mathrm{z}}\right\rangle$. En outre, la sélectivité et la polarisation circulaire nécessitent d'utiliser une source adéquate. Le rayonnement synchrotron répond bien à ces exigences.

Nous avons donc utilisé le dichroïsme magnétique circulaire de RX (souvent appelé XMCD pour XRay Magnetic Circular Dichroïsm) pour étudier le magnétisme de couche ultra-mince de Mn sur Fe(001). L'intérêt d'étudier Mn est le suivant : parmi les éléments 3d, c'est lui qui porte le plus fort moment en atome libre puisque son niveau d est à moitié rempli. En volume, la situation est évidemment différente puisqu'il n'existe plus de niveau d atomique, remplacé par une bande $d$ du métal. Les propriétés magnétiques par rapport à l'atome libre sont ainsi modifiées par l'interaction avec le réseau, et Mn a pour sa part toujours été observé dans des états antiferromagnétiques, aussi bien dans les structures cristallines usuelles comme Mn $\alpha$ et $\operatorname{Mn} \beta$ [3], que dans des phases contraintes ou/et métastables obtenues sur Ag [4], Pd [5] ou Ir [6]. En revanche, un ordre ferromagnétique a été observé récemment sur une monocouche de Mn déposée sur Co 
[7] et dans un alliage de surface MnNi [8]. En ce qưi concerne la croissance sur Fe(001), différentes expériences semblent montrer qu'un ordre ferromagnétique existe à faible recouvrement, alors qu'un ordre antiferromagnétique est clairement démontré au delà de 3 plans atomiques [9]. Enfin, une expérience récente de dichroïsme circulaire [10] a montré qu'une monocouche de Mn est ferromagnétique, avec un couplage antiparallèle entre les moments de Mn et Fe. Nous avons effectué des mesures identiques simultanément, et nous avons obtenu des résultats très différents. Nous exposons ces résultats dans ce papier et après avoir reproduit et examiné les conditions de croissance utilisées dans la ref.10, nous montrons que ces différences proviennent de la préparation de l'échantillon.

\section{DESCRIPTION EXPERIMENTALE}

Les échantillons ont été préparés dans une chambre EJM couplée à la chambre de mesure dichrö̈que. La croissance et la caractérisation des échantillons ont donc été entièrement réalisées sous ultravide, condition indispensable pour toute étude sur le manganèse pur puisqu'il s'oxyde à l'air dans les conditions normales. Les modes de croissance ont été caractérisés par diffraction d'électrons de haute énergie en incidence rasante (RHEED). La qualité chimique des dépôts a été étudiée par spectroscopie Auger et ESCA. Mn a été déposé sur des couches tampons de $200 \AA$ de Fe bcc déposées à l'ambiante sur $\mathrm{MgO}(001)$ et recuites à $600 \mathrm{~K}$. Les couches tampons de fer sont relaxées dans la structure bcc et orientées (001) dans la direction de croissance. La contamination en oxygène des dépôts de Fe et Mn, si elle existe, est inférieure au seuil de détection de l'Auger et l'ESCA, alors qu'une faible contamination en C (estimée au maximum à $4 \%$ ) a été quelques fois observée. Aucune altération des processus de croissance et des propriétés magnétiques des couches n'a en outre été détectée.

Les mesures XMCD ont été réalisées sur la ligne SU2 de l'anneau SuperAco à LURE. Le moment de Fe et Mn étant dans le plan des couches, les mesures XMCD ont été effectuées avec un angle de $70^{\circ}$ entre la normale à l'échantillon et le faisceau $X$ incident. L'aimantation des échantillons est obtenue par l'application d'un champ magnétique dans le plan du substrat. La mesure des seuils est obtenue en mesurant les variations du courant total induit dans l'échantillon pendant les processus d'absorption (technique dite du "total Yield"). Le signal XMCD est obtenu en faisant la différence des seuils $\mathrm{L}_{\text {III-III }}$ de Fe et Mn obtenus non pas en inversant la polarisation circulaire mais en choisissant une polarisation circulaire fixée et en inversant à chaque énergie le sens du champ magnétique appliqué. Le degré de polarisation circulaire est de $70 \%$ et le champ appliqué de 250 Oe sur l'échantillon. Les spectres XMCD montrés ici sont corrigés du degré de polarisation et de l'angle entre les moments et le faisceau $X$.

\section{RESULTATS ET DISCUSSION}

Nous allons tout d'abord donner les résultats concernant la croissance de Mn sur un buffer de Fe épais obtenu par croissance sur MgO. Le RHEED montre que la structure de surface est identique à celle du plan (001) de départ. Des oscillations de RHEED sont observées pendant la croissance de $\mathrm{Mn}$ sur Fe à l'ambiante (fig.1), démontrant ainsi un mode de croissance couche à couche. Parler d'une monocouche de $\mathrm{Mn}$ sur Fe a donc un sens, ce qui ne serait pas le cas si la croissance était tri-dimensionnelle. Une oscillation

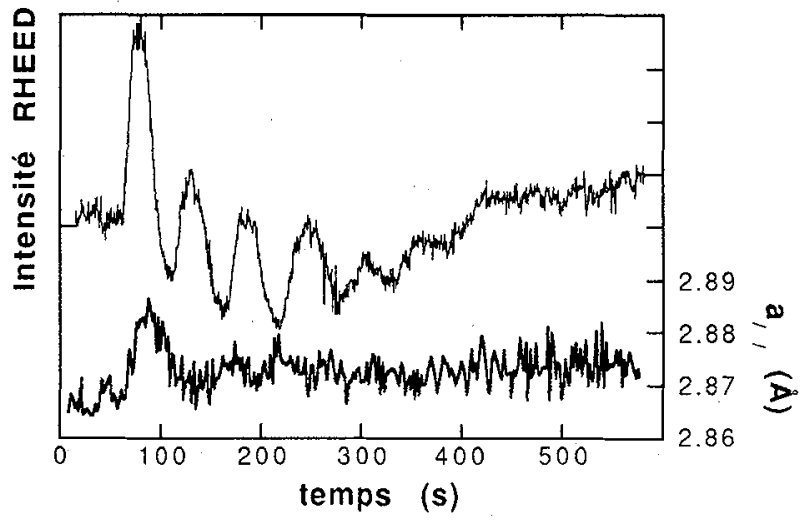

Figure 1: Oscillations de RHEED et variation du paramètre dans le plan pendant la croissance de $M n$ sur Fe 
de RHEED correspond à la croissance d'une couche d'une hauteur atomique, ce qui nous a permis de contrôler précisément les épaisseurs déposées de Mn. L'analyse Auger du pic de $\mathrm{Mn}$ à $542 \mathrm{eV}$ en fonction de la quantité déposée nous a permis de nous assurer qu'il n'y a pas d'interdiffusion de $\mathrm{Mn}$ dans $\mathrm{Fe}$ à l'ambiante. Ceci est en accord avec les résultats de Walker et Hopster [9] qui ont montré que l'interdiffusion est significative au delà de $420 \mathrm{~K}$. Finalement, nous n'avons pas vu de variation significative du paramètre dans le plan de Mn pendant la croissance jusqu'à une dizaine de plans atomiques (fig. 1). La croissance de Mn sur Fe(001) à l'ambiante est donc 2D, sans interdiffusion, et pseudomorphe au plan (001) du fer bec.

Un signal XMCD caractéristique d'un ordre ferromagnétique a été observé jusqu'à deux plans atomiques de Mn. Le couplage interfacial observé entre Mn et Fe est également ferromagnétique (fig.2). De plus, le seuil $\mathrm{L}_{\mathrm{II}-\mathrm{III}}$ de Mn est alors typique d'une transition des niveaux $2 \mathrm{p}$ vers une bande métallique $3 \mathrm{~d}$ (pas d'effet multiplet). Le moment magnétique moyen obtenu en appliquant les règles de somme est de 1 $\mu_{\mathrm{B}} / \mathrm{at}$ à l'ambiante et à $77 \mathrm{~K}$, ce qui veut dire que la température de Curie est bien au delà de l'ambiante. Au delà de 2 monocouches, un signal dichröque peu académique a été observé (fíg.3). Ce type de signal, qui a une forme de signal XMCD dérivé, a en outre déjà été observé dans des alliages ioniques antiferro- ou ferrimagnétiques[12]. En effet, dans ce cas, les niveaux d'énergie entre des sites magnétiques couplés antiparallèlement sont décalés en énergie en raison de l'environnement chimique différent pour les deux sites. Ce type de situation conduit à un signal XMCD provenant de la somme de deux signaux XMCD opposés en signe et décalés en énergie, donc à un signal XMCD classique dérivé. On peut donc expliquer ce type de signal sur Mn comme provenant de l'apparition d'un état antiferromagnétique qui existe effectivement pour les fortes épaisseurs de $\mathrm{Mn}$ [9]. En outre, le décalage des niveaux d'énergie est beaucoup plus difficile à justifier dans les métaux purs. Il semblerait tout de même que ce décalage puisse exister comme le montre un calcul récent de Wu et Freeman [11]. Ce résultat reste cependant à confirmer. Il est néanmoins clair que le changement de signal dichrö̈que correspond à une transition ferro-antiferromagnétique.

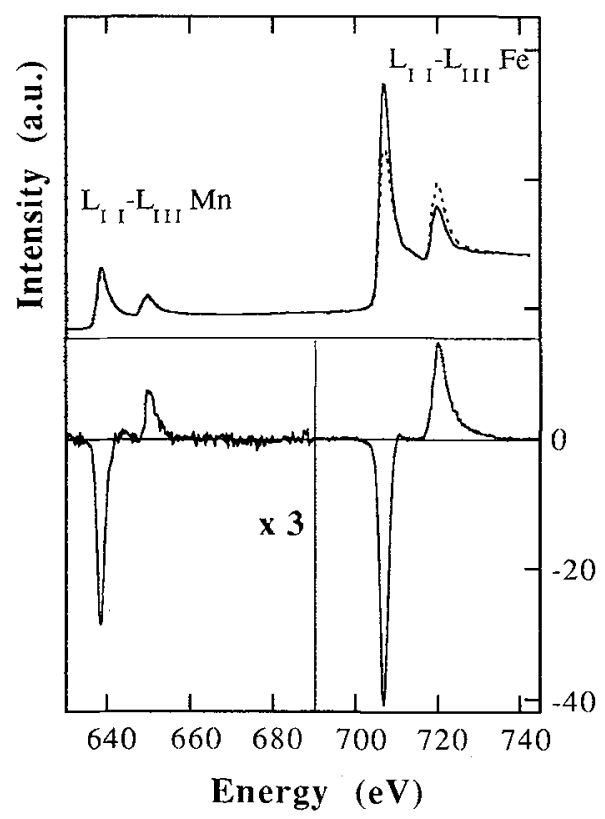

Figure 2: Seuils de Mn et Fe et signal XMCD pour une monocouche de Mn sur Fe(001)

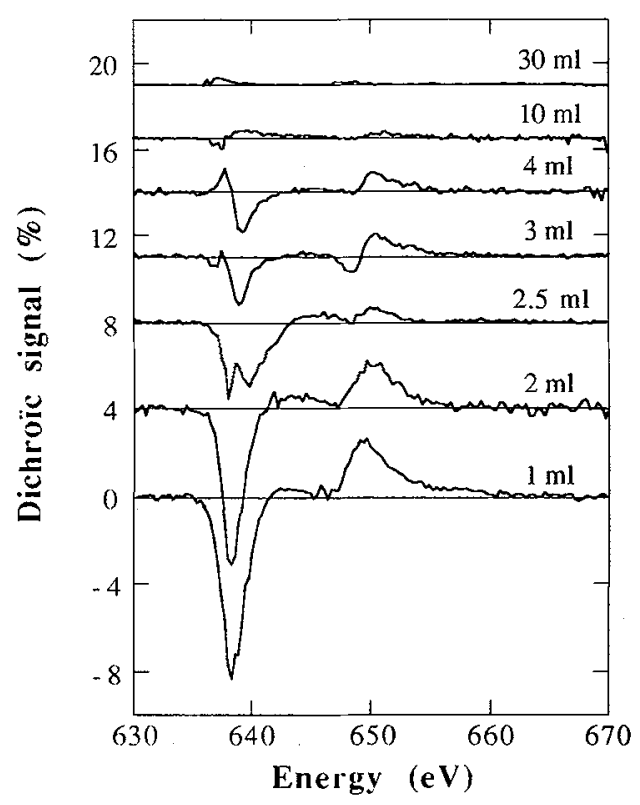

Figure 3 : signal XMCD de Mn sur Fe(00I) en fonction du nombre de plans de Mn déposés

Examinons maintenant le désaccord entre les observations de Dresselhaus et coll. [10] et nos résultats. Nous avons en particulier examiné si la rugosité de surface pouvait jouer un rôle. Les couches tampons de fer déposées sur $\mathrm{MgO}$ et non recuit présentent une rugosité cinétique de l'ordre de $20 \AA$ pour une épaisseur de $200 \AA$. Nous avons déposé une monocouche de Mn sur cette surface rugueuse et examiné ses propriétés magnétiques. Mis à part une diminution de $25 \%$ du moment magnétique, nous avons 
toujours trouvé un comportement ferromagnétique avec un couplage parallèle des moments de $\mathrm{Mn}$ et $\mathrm{Fe}$. Les études en température n'ont également pas montré de changement du sens du couplage. Nous avons alors envisagé l'hypothèse d'une influence éventuelle du mode de préparation du substrat. Nous avons ainsi reproduit l'expérience décrite en [10] : nous avons déposé $20 \AA$ de Fe sur un monocristal d'Ag(001). Nous avons alors observé après le dépôt de Fe un pic intense d'Ag en Auger. Etant donné que la croissance de Fe sur $\mathrm{Ag}(001)$ est bi-dimensionnelle [13], et que $\mathrm{Fe}$ et $\mathrm{Ag}$ ne sont pas miscibles, ce résultat ne peut s'expliquer que par une ségrégation cinétique [14] de Ag sur Fe. Ce résultat n'est somme toute pas surprenant puisque l'énergie de surface d'Ag est environ deux fois plus faible que celle de Fe. Après avoir déposé une monocouche de Mn sur cette surface à l'ambiante, nous avons observé un signal XMCD nul sur $\mathrm{Mn}$. Par contre, en provoquant l'interdiffusion de $\mathrm{Mn}, \mathrm{Ag}$ et $\mathrm{Fe}$ en chauffant jusqu'à $500 \mathrm{~K}$ et en contrôlant en Auger, nous avons alors observé un signal XMCD ferromagnétique sur Mn avec un couplage antiparallèle entre les moments de $\mathrm{Mn}$ et $\mathrm{Fe}$, confirmant ainsi les résultats de Dresselhaus et coll. [10]. Nous montrons ainsi que la couche obtenue n'a rien à voir avec une monocouche de $\mathrm{Mn}$ pure bi-dimensionnelle.
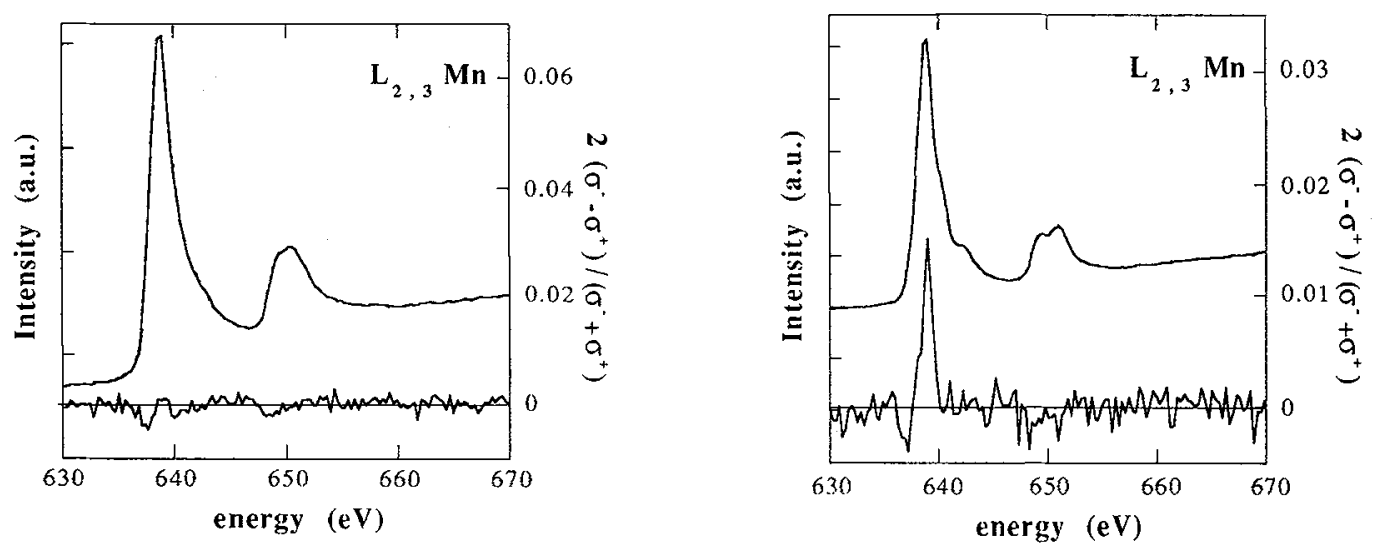

\section{Figure 4 : Seuils et spectres XMCD obtenus pour une monocouche de Mn déposée sur $20 A ̊ F e l A g(001)$ avant (à gauche) et après recuit (à droite)}

\section{CONCLUSION}

En résumé, nous avons montré que les contradictions apparentes entre les résultats de la ref.[10] et ceux rapportés ici proviennent de la préparation de l'échantillon. Un examen approprié des conditions de croissance nous a permis de montrer que les résultats reportés en [10] correspondent à du manganèse interdiffusé dans Ag. En revanche, nous avons montré que notre préparation des échantillons nous permet effectivement bien d'obtenir une monocouche bi-dimensionnelle de Mn pseudomorphe au plan (001) de Fe. Nous montrons ainsi définitivement que le couplage interfacial entre les moments de $\mathrm{Mn}$ et $\mathrm{Fe}$ est ferromagnétique.

Nous avons également observé que le manganèse est ferromagnétique jusqu'à deux plans atomiques, mais le moment obtenu est beaucoup plus faible que le laissent prévoir les diverses modélisations $[11,15]$. Plusieurs explications sont possibles. Tout d'abord, on peut remettre en cause la validité des règles de somme dans le cas de $\mathrm{Mn}$, car le couplage spin-orbite conduisant à la séparation des niveaux $2 \mathrm{p}^{1 / 2}$ et $2 \mathrm{p}^{3 / 2}$ est alors trop faible pour justifier les approximations conduisant à ces règles. Néanmoins, un calcul récent de Wu et Freeman [11] dans le cas d'une monocouche de Mn sur Fe avec un moment de $3 \mu_{\mathrm{B}} /$ at donnent un signal XMCD 3 fois plus grand que celui observé ici, ce qui confirme la valeur de $1 \mu_{\mathrm{B}} /$ at. Il est également possible que le volume atomique utilisé dans les calculs ne soit pas réaliste. Le paramètre dans le plan de Mn est bien celui du fer, comme nous l'avons montré ici. Par contre, le paramètre entre plans choisi est celui obtenu par Purcell et coll. [9] dans des superréseaux $\mathrm{Mn} / \mathrm{Fe}(001)$ avec des épaisseurs de Mn supérieures à deux monocouches. Rien ne justifie à priori que cette distance ne varie pas au moment de la transition ferro-antiferromagnétique. Pour vérifier ce point, nous avons effectué des expériences d'EXAFS in situ qui sont en cours d'analyse. 


\section{Références}

[1] Voir par exemple la thèse de J.P. Schillé, Université de Strasbourg, 1994

[2] Carra P. et al, Phys. Rev. Lett., 70, (1993), 694

[3] Sliwko V. et al, J. Phys. Cond. Matter, 6, (1994), 6557

[4] Jonker B.T. et al, Phys. Rev. B, 39, (1989), 1399.

[5] Tian D. et al, Solid State Com., 70, (1989), 199.

[6] Andrieu S. et al, Phys. Rev. B, 54, (1996), 2822.

[7] O'Brien W.L. et Tonner B.P., Phys. Rev. B, 50, (1994), 2963.

[8] O'Brien W.L, et Tonner B.P., Phys. Rev. B, 51, (1995), 617.

[9] Purcell S.T, et al, Phys. Rev. B, 45, (1992), 13064

Walker T.G. et Hopster H., Phys. Rev. B, 48, (1993), 3563

Roth Ch.et al, Phys. Rev. B, 52, (1995), 15691

[10] Dresselhaus J. et al, E-MRS, 4-7 juin 1996, Strasbourg (France), J.Mag.Mag.Mat., sous presse

[11] Wu R.Q. et Freeman A.J., Phys. Rev. B, 51, (1995), 17131

Attention, ces auteurs définissent le signal XMCD comme $\left(\sigma^{+}-\sigma^{-}\right) / \sigma_{\mathrm{t}}$ avec $\sigma_{\mathrm{t}}=\sigma_{0}+\sigma^{+}+\sigma^{-}$. En pratique on ne mesure pas $\sigma_{0}$ car $\sigma_{0}=\left(\sigma^{+}+\sigma^{-}\right) / 2$ et le signal XMCD est défini ici par $2\left(\sigma^{+}-\sigma^{-}\right) /\left(\sigma^{+}+\sigma^{-}\right)$.

[12] Sette F. et al, dans X-Ray Absorption Fine Structure, édité par Hasnain S.S. (Ellis Howood, N.Y., 1991) p.96

[13] Heinrich B. et al, Phys. Rev. B, 38, (1988), 12879

[14] Andrieu S. et al, J. Appl. Phys., 65, (1989), 2681

[15] Mbirt S. et al, Phys. Rev. B, 52, (1995), 15070

Bouarab S, et al, Phys. Rev. B, 52, (1995), 10127

Vega A. et al, Thin Solid Films, 275, (1996), 103 